\title{
High-resolution comparative chromosome painting in the Arizona collared peccary (Pecari tajacu, Tayassuidae): a comparison with the karyotype of pig and sheep
}

Filomena Adega ${ }^{1}$, Raquel Chaves ${ }^{1 *}$, Andrea Kofler ${ }^{2}$, Paul R. Krausman ${ }^{3}$, Julio Masabanda ${ }^{4}$, Johannes Wienberg ${ }^{2,5}$ \& Henrique Guedes-Pinto ${ }^{1,3}$

${ }^{1}$ Department of Genetics and Biotechnology, Centre of Genetics and Biotechnology Y CGB, University of Trásos-Montes and Alto Douro Y UTAD, 5001-801, Vila Real, Portugal; Tel: +351-259-350571; Fax: +351- 259-

350572; E-mail: rchaves@utad.pt;

${ }^{2}$ Chrombios GmbH, Mühlenstr.1, D-83064, Raubling, Germany;

${ }^{3}$ School of Natural Resources, University of Arizona, Tucson, AZ 85721, USA; ${ }^{4}$ Department of Biosciences, University of Kent, Canterbury, Kent CT2 7NJ, UK; ${ }^{5}$ Department of Biology II, University of Munich, Großhaderner Strasse 2, 82152, Planegg-Martinsried, Germany

* Correspondence

Key words: Cetartiodactyla, chromosome rearrangements, Karyotype evolution, Pecari tajacu, Suiformes

\begin{abstract}
We used chromosome painting with chromosome-specific probes derived from domestic sheep and pig for a high-resolution cytogenetic comparison with the karyotype of collared peccary (Pecari tajacu sonoriensis). A reorganization of the karyotype involving at least $62 \mathrm{Y} 66$ conserved segments were observed between the sheep and collared peccary. This is an extremely high number compared with other members of the same mammalian order (Cetartiodactyla). The comparison between pig and collared peccary, both belonging to the Suiformes, however, revealed various changes in the gross organization of both karyotypes that may have already occurred in a common ancestor of both species suggesting a monophyletic origin of Suidae/Tayassuidae. The sheep probes, however, also revealed several rearrangements between the two Suidae/Tayassuidae, indicating that these probes represent a useful tool for a more detailed analysis of the evolutionary history of Suiformes. Our sample of the collared peccary from North America (Arizona, USA) showed distinct differences to those already described from South America. The chromosome painting results defined a complex translocation that involves chromosomes including about one-quarter of the entire collared peccary karyotype. This considerable rearrangement indicates subspecies or even species status of both peccary populations, as it should present a significant barrier for their hybridization.
\end{abstract}

Introduction

Peccaries (or Fpecari_, a Brazilian Tupi word for Fan animal which makes many paths through the woods_) also known as Tayassuidae (Fgnawers of roots_) or Dycotilidae, are endemic Suiformes of the Americas. In North America their known fossil record extends back to the late Eocene, although there are some purported fossil remains of peccaries from the late Eocene through the late Miocene found in Eurasia and Africa (Groves \& Grubb 1993). The Tayassuidae diverged from the Suidae, the Ftrue pigs_, nearly 40 million years ago. Since then the peccary phylogeny has split into various lines leading to three recog- 
nized extant species: the collared peccary [Pecari (syn. Tayassu, Dycotiles) tajacu], ranging from North AmericaYArizona, New Mexico and Texas to South America, the white-lipped (Tayassu pecari) and the Chacoan peccaries (Catagonus wagneri). The later two species are thought to be close relatives and found in more tropical South American areas. The Chacoan peccary is one of the most recently described mammals; it was reported from the Paraguayan Chaco Valley in 1975 (Groves \& Grubb 1993).

Over the past 15 years chromosome painting has been extensively used in cytotaxonomy and phylogenetic studies (see recent reviews by Wienberg 2004, 2005, Froenicke 2005). When hybridizing fluorochrome-labeled chromosome-specific DNA probes of one species to the chromosomes fixed on slides of another species, homologies can be identified at the microscope between both species that are based on homologous DNA sequences. Thus, in recent years this technique has allowed us to establish various gross comparative homology maps between and within most mammalian orders (O'Brien et al. 1999a, 1999b).

Recently, Bosma et al. (2004) used chromosome painting to establish homology maps between the domestic pig, collared and white-lipped peccaries using pig painting probes on peccary chromosomes. The peccaries analyzed by these authors were of South American origin but the exact locale was uncertain. Their work revealed dramatic changes of Tayassuidae karyotypes when compared to the pig. There were, however, also extensive karyotypic differences between the two Tayassuidae species analyzed.

Here we used both pig and sheep chromosome painting probes for a high-resolution cytogenetic comparison between sheep, pig and a population of collared peccary from North America (Arizona, USA) that, when compared with the already-described sample from South America (Bosma et al. 2004), showed distinct differences. The use of painting probes from a high chromosome number cetartiodactyl karyotype (sheep, $2 \mathrm{n}=54$ ) to one of a low chromosome number (collared peccary, $2 \mathrm{n}=30$ ), allowed the delineation not only of translocations, but also various inversions that occurred in the phylogenetic lines of these species. The chromosome painting results define a complex translocation in the karyotype of the Arizona population compared to the South American specimens, which may have a serious taxonomic impact requiring a change in taxonomy.
Materials and methods

Cell culture and chromosome preparation

Metaphase preparations from a total of eight collared peccary (Pecari tajacu sonoriensis PTA) individuals from different regions of Arizona, USA, kindly supplied by Tucson Wildlife Center, Inc. were prepared from short-term lymphocyte cultures of peripheral blood samples using standard protocols (Chaves et al. 2002).

In general, for karyotyping, peccary chromosomes were ordered according to Bosma et al. (2004) into metacentric, submetacentric and acrocentric chromosomes and according to the relative size of PTAar chromosomes. Because the karyotype of our samples differs from the one described by Bosma et al. (2004), we slightly adapted the chromosome numbering (see Figure 3). Chromosome numbering of pig (Sus scrofa, SSC) followed the Committee for the Standardized Karyotype of the Domestic Pig (1988). Chromosome numbering for sheep (Ovis aries, OAR) paint probes was the same as described previously (Burkin et al. 1997).

Chromosome painting probes and probe labeling

Flow-sorted pig chromosome-specific probes were kindly provided by the Cambridge Resource Centre for Comparative Genomics and D. Griffin, University of Kent (UK). Sheep chromosome-specific probes were the same as described by Burkin et al. (1997). Chromosome-specific probes were made by degenerate oligonucleotide primed PCR (DOP-PCR) from the flow-sorted chromosomes by using 6MW-PCR primers and amplification conditions as previously described (Rabbitts et al. 1995). In a secondary PCR the same PCR primers were also used to label the chromosome paints with haptens or fluorescentlabeled dUTP.

Sheep probes were labeled with digoxigenin-dUTP or biotin-dUTP (Roche Molecular Biochemicals). When analyzing pig/peccary homologies, four pools of pig paint probes were used, each consisting of five different chromosomes labeled with different haptens or fluorescence-labeled dUTP. Pig probes were labeled with DEAC-dUTP (NEN), digoxigenin-dUTP and biotin-dUTP (Roche Molecular Biochemicals), TexasRed-dUTP (Molecular Probes), and Cy3-dUTP (Amersham). 
Fluorescent in-situ hybridization and image processing

In-situ hybridization of sheep painting probes to $\mathrm{T}$. tajacu metaphase spreads was done as previously described (Wienberg et al. 1997) and after hybridization and washing of the slides, digoxigeninlabeled chromosome paints were detected with rhodamine-labeled goat-anti-digoxigenin antibodies (Roche Molecular Biochemicals), and biotin-labeled probes were detected with avidin-FITC (Vector Laboratories).

For the pig multicolor painting on peccary chromosomes digoxigenin-dUTP labeled paints were detected with anti-dig-FITC-antibodies (Roche Molecular Biochemicals) and biotin-labeled probes were detected with Avidin-Cy5 (Amersham). DEAC, Cy3 and Texas Red-labeled paints did not need any detection procedures because they were directly labeled with fluorochrome-coupled dUTP (see above).

Digital images were obtained with an Axiocam or Photometrics Sensys camera coupled to a Zeiss Axioplan 2 microscope and analyzed by AxioVision software (Zeiss) or SmartCapture VP software (Digital Scientific, Cambridge, UK), respectively. Fluorescent filters for multicolor painting with pig probes were the same as described by Fauth \& Speicher (2001).

\section{Results}

The karyotype of the eight collared peccaries from Arizona, USA (PTAar) had 2n $=30$ (Giannoni et al. 1981) chromosomes, which is the same chromosome number as the South American samples analyzed by Bosma et al. (2004). Because the exact origin of the later individuals was unknown except that they were from Brazil, we term them PTAbr. Figure 3 shows the karyotype for G-banded PTAar chromosomes. The chromosome numbering for the PTAbr sample is given in brackets. By simply inspecting the chromo- some forms there are obvious differences between the two samples: in PTAar the autosomes consist of a large metacentric pair, one large submetacentric, six medium submetacentric and six smaller submetacentric/acrocentric chromosomes. The sex chromosomes consist of a medium submetacentric $\mathrm{X}$ and a small submetacentric $\mathrm{Y}$ that is the smallest chromosome of the karyotype. The PTAbr sample described by Bosma et al. (2004) also consisted of a large metacentric pair that, however, was significantly larger, and one large submetacentric. In the PTAbr karyotype there are five instead of six medium submetacentrics and seven instead of six smaller submetacentric/acrocentric chromosomes, compared to the Arizona samples. Further, in the PTAbr karyotype, the $\mathrm{X}$ chromosome is acrocentric while the PTAar presents a submetacentric $\mathrm{X}$ similar to most of other mammals.

For a high-resolution comparison, sheep paints of all individual chromosomes were in-situ hybridized to Arizona collared peccary chromosomes. The hybridization results are summarized in Figure 3, by a map of the hybridization sites on the haploid karyotype of this peccary sample. Figure 1 shows various examples for hybridization images obtained with sheep painting probes. Sheep chromosome paints revealed an unusually high number of chromosomal rearrangements between these two species. The sheep paint probes disclosed at least 64 painted segments in the collared peccary. The Y chromosome was the only sheep paint probe that failed in providing a specific hybridization signal.

There were only two sheep (OAR) paint probes that delineated one homologous peccary (PTAar) chromosome only: OAR14 and OAR X hybridized to PTAar11 and PTAar X, respectively. The probes derived from OAR13, OAR16, OAR19, OAR22 and OAR25 each provided only one signal in some chromosomes of the collared peccary, that were, however, further rearranged (Figures 1 and 3). All the other sheep paint probes showed homologies to two or more segments in one or more chromosomes of the collared peccary. Some of the collared peccary autosomes proved to be composed of more than eight and up to 11 different homologous segments compared to the sheep (i.e. PTAar chromosomes 1, 2 and 3). Collared peccary chromosome 1 displayed a puzzle of hybridization signals of at least 17 sheep conserved segments consisting of 11 different sheep chromosome probes (OAR2, OAR7, OAR9, OAR12, OAR17, OAR20, OAR22, OAR23, OAR24, OAR25 and OAR26). For some few small regions at collared peccary chromosomes PTAar1, PTAar2, PTAar5, PTAar7 and PTAar10 hybridization signals were too complex to define an exact homology pattern (indicated with an F?_ in Figure 3). 
Most interestingly, however, the sheep paints displayed in detail the differences between the karyotypes of our samples of collared peccary PTAar and the PTAbr sample described by Bosma et al. (2004). The short arm of a medium-sized meta/ submetacentric (PTAar5) chromosome, which is not found in the PTAbr sample, consists of a segment homologous to a large block in PTAbr chromosome 1qter. The long arm of PTAar5 is homologous to an acrocentric chromosome (chromosome 11 in PTAbr karyotype), not present in our sample. Compared to the South American sample this would explain the change of the karyotype of the Arizona sample as a fusion of an acrocentric chromosome with a segment from the large chromosome 1, adding one more metacentric and losing an acrocentric without any change in the chromosome number. This description should not indicate any direction of changes because without any appropriate outgroup species it could be a fission and fusion or vice versa.

For a better confirmation of the sheep/peccary homology map, chromosome painting was also performed with pig chromosome-specific probes on the sample from the collared peccary. Chromosome painting with four pools, each consisting of five different pig paint probes, was used to delineate chromosome changes between the pig and the PTAar karyotypes. One paint (pig chromosome 15) was used twice in two different pools. To simplify the analysis we used multicolor chromosome painting, and each pig paint in an individual pool got a Funique label_ (haptens or directly labeled dUTP) that was analyzed through very narrow fluorescent bandpass filters. This procedure allowed a simple assignment of each probe without the need to establish mixed probe sets in Boolean combinations as described by Ried et al. (1992). Larger probe sets labeled in Boolean combinations as described for cross-species hybridization in some primates (Schröck et al. 1996) were not working between pig and peccary in the present experiments.

Each pool of pig paints delineated distinct segments of peccary chromosomes summarized in Figure 3. A hybridization image of pool 1 is given in Figure 2 as an example. Since these experiments were done independently from the painting experiments with sheep probes in two different laboratories (Vila Real, Munich) without detailed knowledge of the respective results, the outcome is convincing: nearly all homologies defined by the more complex sheep paint probes were confirmed and extended by the pig chromosome painting. A few discrepancies (minor signals from pig chromosome 6 and 10 paints) were resolved by further hybridizations using single pig/sheep chromosome painting probes.

\section{Discussion}

The current analysis allowed us to establish a highresolution comparative cytogenetic map between sheep, pig, and the North American Arizona population of the collared peccary. The chromosome painting data confirm and extend previous findings showing that the main differences between pig and peccary chromosomes are translocations and fusion/ fission of large chromosome segments (Bosma et al. 2004). Further, the data also confirm and extend assumptions that the pig has a highly derived karyotype compared to other Cetartiodactyla (Schmitz et al. 1998, Froenicke \& Wienberg 2001), and to primates (humans), serving as an outgroup (Froenicke et al. 1996, Goureau et al. 1996, Schmitz et al. 1998, Pinton et al. 2003, Biltueva et al. 2004).

Suiformes are believed to have diverged very early from the main stem of cetartiodactyls (Groves \& Grubb 1993). Obviously, representatives of later branches, such as the Ruminantia and especially the Cetacea (i.e. whales and dolphins) conserved much more of the ancestral karyotype of all placental mammals, while early Suiformes experienced drastic reshuffling of their karyotype (Bielec et al. 1998, Froenicke \& Wienberg 2001, Biltueva et al. 2004, Froenicke 2005). Bovid karyotypes, although changed by many centromeric fissions, conserved most motifs in chromosome morphology, that can easily be reconstructed from the ancestral karyotype of all cetartiodactyls and even from that of most other mammals (Chowdhary et al. 1998, Wienberg et al. 2000, Froenicke 2005).

Froenicke \& Wienberg (2001) used sheep chromosome paints on pig chromosomes for a high-resolution cytogenetic map between these two artiodactyl species and revealed numerous inter-chromosomal rearrangements that should have occurred in suid phylogeny. The high number of small sheep chromosomes $(2 \mathrm{n}=54)$ compared to the in general larger pig chromosomes $(2 n=38)$ made it possible to use these paints also as subregional probes and to delineate various intra-chromosomal rearrangements 
in the pig karyotype (Froenicke \& Wienberg 2001). This approach has been successfully used in other species to define subregional homologies between and within members of various mammalian taxa (Yang et al. 1999, Müller et al. 2000, Murphy et al. 2003, Biltueva et al. 2004).

In the previously published pig/sheep comparative map, 62 conserved segments were observed (Froenicke \& Wienberg 2001). In our present experiments we also were able to define at least $62 Y 66$ conserved segments between sheep and peccary, representing extremely high numbers when comparing species within the same mammalian order. With sheep paints, many of these segments have the same pattern in pig and collared peccary. These results indicate that the great difference of the karyotypes of these species is due to reshuffling of entire chromosomes or large chromosome segments, and that the main reshuffling took place in a common ancestor of pig and peccaries. Some patterns of sheep paintings (adjacent painting patterns) in the collared peccary, however, were apparently absent from the pig karyotype: these include associations of signals derived from sheep (OAR) chromosomes 8/17, 1/3 1/10, $1 / 15,24 / 2 / 7,20 / 2,26 / 3,4 / 16,5 / 11,7 / 11,11 / 23$, $12 / 24,21 / 4,22 / 20,24 / 9,24 / 2$, and $26 / 1$ that may be due to various new fusion products, or other derived rearrangements in the peccary. Other sheep painting patterns indicate inversions. In the pig karyotype, sheep painting probes delineated nine inversions (Froenicke \& Wienberg 2001). Six of them are also evident in the peccary and should be considered as common derived traits of both species (OAR2/26 on PTAar1/SSC15; OAR7/23 on PTAar1/SSC1; OAR5/7 on PTAar3/SSC2; OAR1/19 on PTAar2/SSC13; OAR7/18 on PTAar7/SSC7 and OAR15/21 on PTAar8 and PTAar9/SSC2 and SSC9, Figure 3). Three further inversions, however, are specific for the peccary (OAR3/24 on PTAar5, 24/9 on PTAar 1 and 3/26 on PTAar2). Yet the wealth of chromosome rearrangements delineated with the sheep probes indicates that they represent a useful tool for a more detailed analysis of the evolutionary history of Suiformes.

The comparative chromosome map of the South American collared peccary previously published by Bosma et al. (2004), established with pig paint probes, revealed 31 conserved segments. We found 32 Y35 conserved segments between the collared peccary from Arizona and pig, and the painting pattern seems very similar. In addition to the collared peccary, Bosma et al. (2004) also analyzed the whitelipped peccary ( $\mathrm{T}$. pecari, $2 \mathrm{n}=26$ ) with pig paints.
The number of conserved segments between this species and pig was in the same range, which is typical for closely related species. The G-banded karyotype of the recognized remaining extant peccaries species, the Chacoan peccary (Catagonus wagneri, $2 \mathrm{n}=20$ ), suggests that this species may be more rearranged. Painting with sheep probes may reveal further insights into the phylogenetic relationship of these species.

The present chromosome painting results also allowed us to identify the difference in the two karyotypes observed in the collared peccary samples from Arizona and South America. Among different populations of white-lipped peccary from Brazil, differences in the $\mathrm{X}$ chromosome centromere position were already reported with banded chromosomes (Giannoni et al. 1981). The Arizona sample described here has an $\mathrm{X}$ chromosome that is more typical for a mammalian X. Although both karyotypes of the two collared peccaries have 30 chromosomes, the present analysis can distinguish both samples by a complex chromosome rearrangement that would involve several chromosomes: a segment at PTAbr1qter and chromosome PTAbr11 together form a metacentric chromosome in the PTAar sample (PTAar5, Figure 3). In meiotic pairing of chromosomes in a hypothetical hybrid between individuals of the two Fpopulations, we would expect a multivalent including four chromosomes representing about one-quarter of the entire peccary genome. Due to multiple unbalanced segregation products, this complex rearrangement should represent an effective barrier in the hybridization of both populations, and confirms the subspecies status given to the morphologically different collared peccary populations, and may even suggest different species.

Recently cytogenetic analyses have revealed instances where dramatic chromosome rearrangements suggest new subspecies or even species in cases where no significant differences in phenotypes were evident. In various primate species, Fpopulations_ have been described for which fertile hybrids have never been recorded, or would be highly unlikely from the complex differences in their karyotypes (Wienberg 2005, for recent review). Some howler monkeys (Alouatta), which were traditionally classified as subspecies, recently achieved species status by chromosome painting analysis (Consigliere et al. 1996). Thus, chromosome painting represents a valuable tool for unrevealing hidden subspecies and species where morphology, molecular data and other traits do not provide sufficient clues. The analysis by chro- 
mosome painting of further collared peccary populations from North and South America will give more insight into their evolutionary history.

\section{Acknowledgements}

We thank the Cambridge Resource Center for Comparative Genomics and D. Griffin, University of Kent (UK) and M.A. Ferguson-Smith and D. Burkin for providing the pig and the sheep painting probes, respectively; Lisa Bates from Tucson Wildlife Center, Inc. and Donald DeYoung from the University of Arizona, for their assistance in the peccaries blood collection. This work was supported by a $\mathrm{PhD}$ grant (SFRH/BD/3280/2000 from the Science and Technology Foundation Y FCT Y from Portugal), INVOTAN and FCT (SFRH/BSAB/329/2003) sabbatical grants.

\section{References}

Bielec PE, Gallagher DS, Womack JE, Busbee DL (1998) Homologies between human and dolphin chromosomes detected by heterologous chromosome painting. Cytogenet Cell Genet 81: $18 Y 25$.

Biltueva LS, Yang F, Vorobieva NV, Graphodatsky AS (2004) Comparative map between the domestic pig and dog. Mamm Genome 15: 809Y818.

Bosma AA, de Haan NA, Arkesteijn GJ, Yang F, Yerle M, Zijlstra C (2004) Comparative chromosome painting between the domestic pig (Sus scrofa) and two species of peccary, the collared peccary (Tayassu tajacu) and the white-lipped peccary (T. pecari): a phylogenetic perspective. Cytogenet Genome Res 105: 115 Y121.

Chowdhary BP, Raudsepp T, Fronicke L, Scherthan H (1998) Emerging patterns of comparative genome organization in some mammalian species as revealed by Zoo-FISH. Genome Res 8: 577 Y589.

Consigliere S, Stanyon R, Koehler U, Agoramoorthy G, Wienberg J (1996) Chromosome painting defines genomic rearrangements between red howler monkey subspecies. Chromosome Res 4: 264Y270.

Fauth C, Speicher MR (2001) Classifying by colors: FISH-based genome analysis. Cytogenet Cell Genet 93: 1Y10.

Froenicke L (2005) Origins of primate chromosomes Yas delineated by Zoo-FISH and alignments of human and mouse draft genome sequences. Cytogenet Genome Res 108: 122Y138.

Froenicke L, Wienberg J (2001) Comparative chromosome painting defines the high rate of karyotype changes between pigs and bovids. Mamm Genome 12: 442 Y449.

Froenicke L, Chowdhary BP, Scherthan H, Gustavsson I (1996) A comparative map of the porcine and human genomes demon- strates ZOO-FISH and gene mapping-based chromosomal homologies. Mamm Genome 7: 285 Y290.
Goureau A, Yerle M, Schmitz A et al. (1996) Human and porcine correspondence of chromosome segments using bidirectional chromosome painting. Genomics 36: 252 Y262.

Groves CP, Grubb P (1993) The Suborder Suiformes. In: Oliver, WLR, ed. Pigs, Peccaries, and Hippos. Status Survey and Conservation Action Plan. Switzerland: International Union for Conservation of Nature and Natural Resources.

Müller S, Stanyon R, Finelli P, Archidiacono N, Wienberg J (2000) Molecular cytogenetic dissection of human chromosomes 3 and 21 evolution. Proc Natl Acad Sci USA 97: 206Y211.

Murphy WJ, Froenicke L, O'Brien SJ, Stanyon R (2003) The origin of human chromosome 1 and its homologs in placental mammals. Genome Res 13: 1880Y1888.

O'Brien SJ, Eisenberg JF, Miyamoto M et al. (1999a) Genome maps 10. Comparative genomics. Mammalian radiations. Wall chart. Science 286: 463 Y478.

O'Brien SJ, Menotti-Raymond M, Murphy WJ et al. (1999b) The promise of comparative genomics in mammals. Science 286: 458 Y 481 .

Pinton A, Ducos A, Yerle M (2003) Chromosomal rearrangements in cattle and pigs revealed by chromosome microdissection and chromosome painting. Genet Sel Evol 35: 685Y696.

Ried T, Baldini A, Rand TC, Ward DC (1992) Simultaneous visualization of seven different DNA probes by in situ hybridization using combinatorial fluorescence and digital imaging microscopy. Proc Natl Acad Sci USA 89: 1388Y1392.

Schmitz A, Oustry A, Vaiman D, Chaput B, Frelat G, Cribiu EP (1998) Comparative karyotype of pig and cattle using whole chromosome painting probes. Hereditas 128: 257 Y 263.

Schrö ck E, du Manoir S, Veldman T et al. (1996) Multicolor spectral karyotyping of human chromosomes. Science 273: 494Y497.

Wienberg J (2004) The evolution of eutherian chromosomes. Curr Opin Genet Dev 14: 657Y666.

Wienberg J (2005) Fluorescence in situ hybridization to chromosomes as a tool to understand human and primate genome evolution. Cytogenet Genome Res 108: 139Y160.

Wienberg J, Frönicke L, Stanyon R (2000) Insights into mammalian genome organization and evolution by molecular cytoge- netics. In: Clark MS, ed. Comparative Genomics. Dordrecht: Kluwer, pp. 207 Y244.

Yang F, O'Brien PC, Milne BS et al. (1999) A complete comparative chromosome map for the dog, red fox, and human and its integration with canine genetic maps. Genomics 62: 189Y202. 


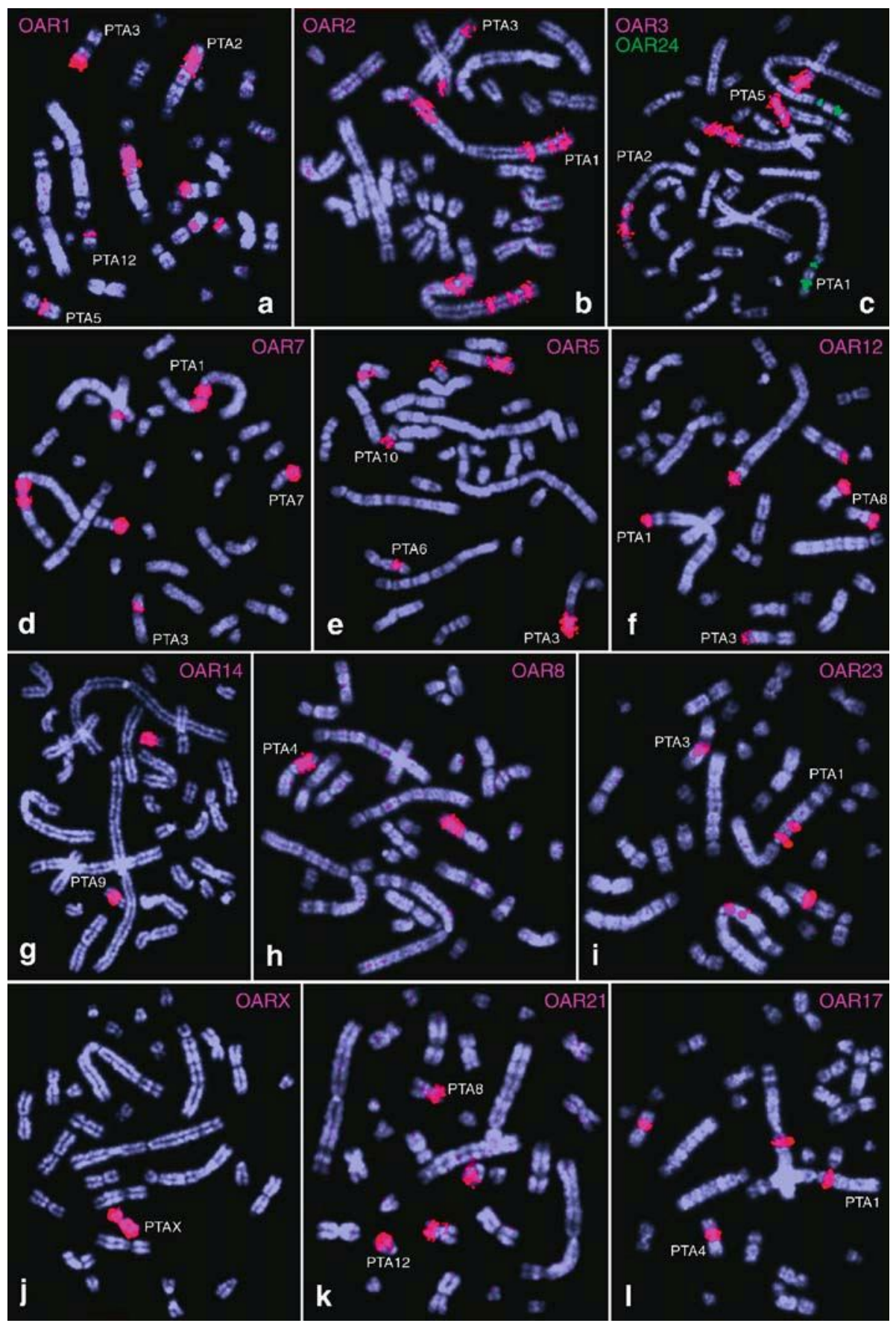

Figure 1. Representative FISH signals of cross-species in-situ hybridization experiments with sheep (OAR) chromosome-specific painting probes to Arizona collared peccary (PTA) chromosomes (aYl). The paint probes are indicated in the corner of each picture segment. The target chromosomes are numbered close to the hybridization signals. 

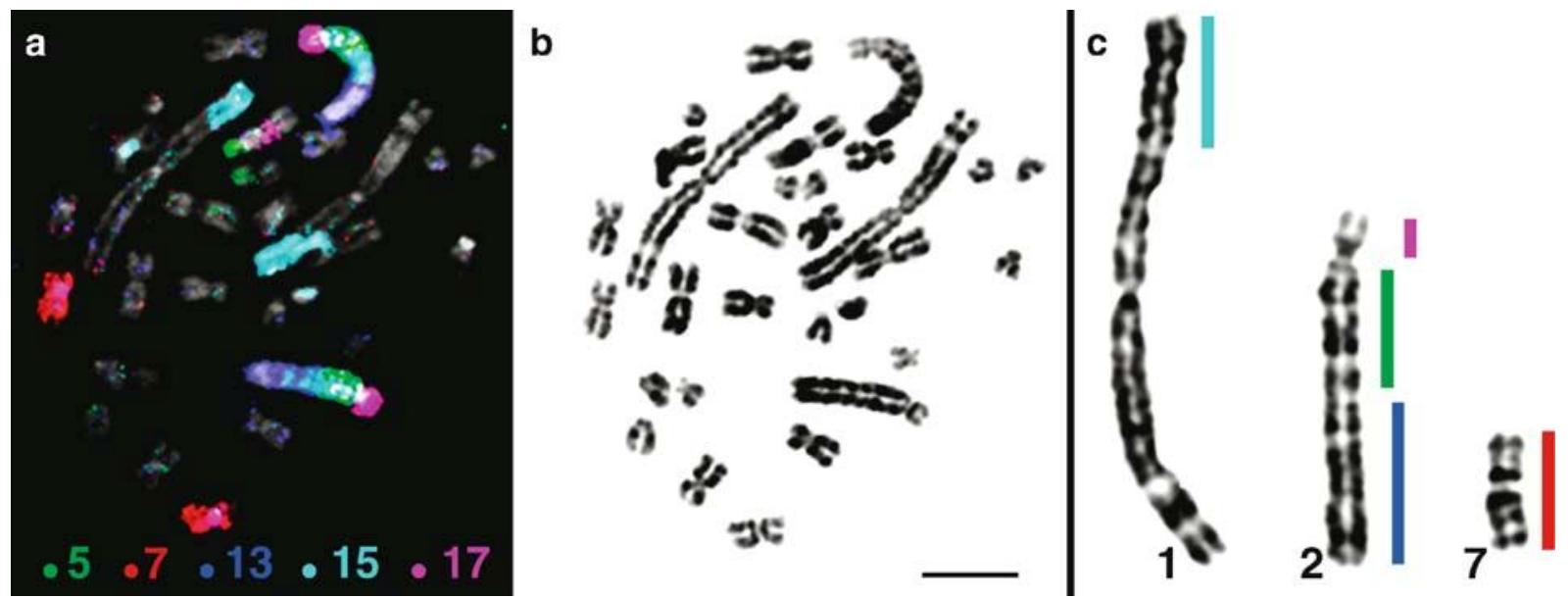

Figure 2. (a) Example of an in-situ hybridization experiment in a multicolor format with a five chromosome-specific pig probes pool (SSC5, 7, 13, 15 and 17) on the Arizona collared peccary chromosomes. Note considerable unspecific hybridization noise to smaller chromosomes which is common in multicolor FISH experiments when comparing species that have diverged for more than 40 million years. (b) DAPI banding of the previous image. (c) The colored lines indicate the homologies detected with the pig paints in the peccary chromosomes PTA1, 2 and 7. (Scale bar $=10$ micrometers.) 

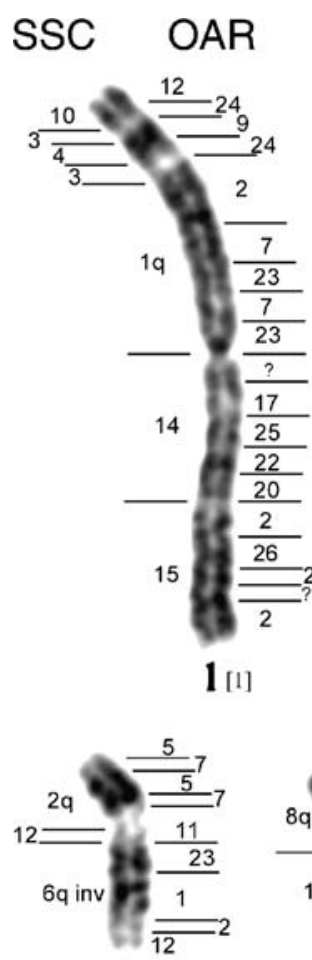

3 [3]

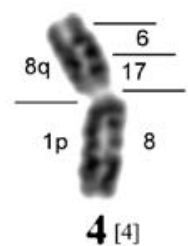

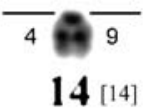
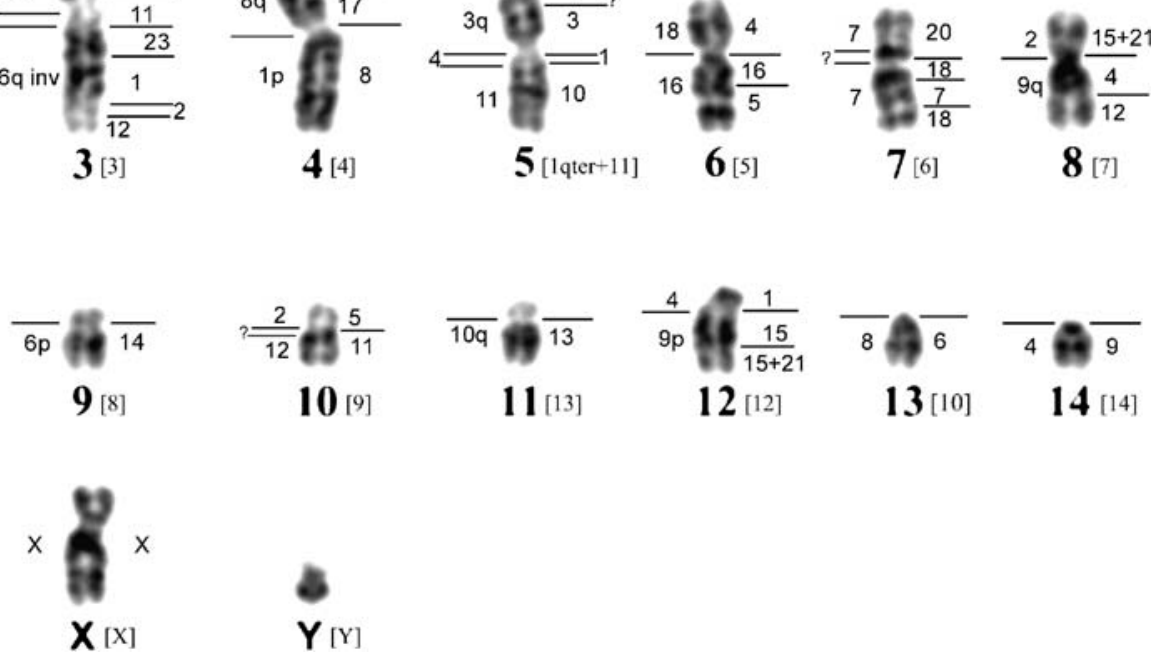
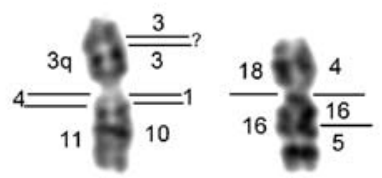

5

6 [5]
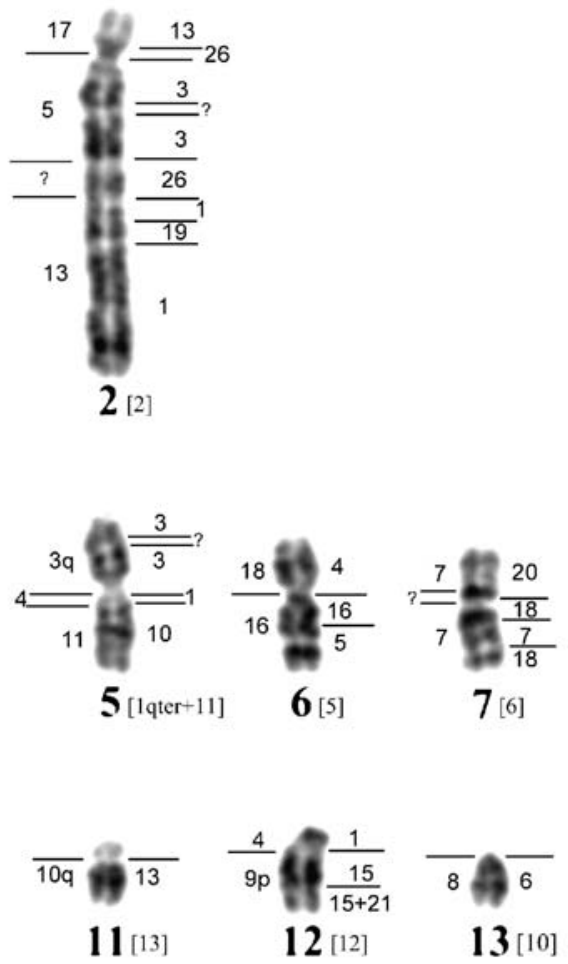

$14[14]$

Figure 3. Comparative genome map of the collared peccary from Arizona (PTAar) displaying the chromosomal homologies to sheep and domestic pig chromosomes in the haploid karyotype of the GTG-banded PTAar chromosomes. The homologies delineated by individual sheep paint probes are numbered at the right and the chromosomal segments hybridized by the pig paint probes at the left of each collared peccary chromosome. For a better comparison between the PTAar and PTAbr chromosomes, the chromosome numbering for the PTAbr sample is given in brackets. 